\title{
GEOGRAPHIC ENVIRONMENTAL KUZNETS CURVES: THE OPTIMAL GROWTH LINEAR-QUADRATIC CASE
}

\author{
Raouf Boucekkine ${ }^{1}$, Giorgio Fabbri ${ }^{2, *}$, SAlvatore Federico ${ }^{3}$ \\ AND FAUsto GozzI ${ }^{4}$
}

\begin{abstract}
We solve a linear-quadratic model of a spatio-temporal economy using a polluting oneinput technology. Space is continuous and heterogenous: locations differ in productivity, nature selfcleaning technology and environmental awareness. The unique link between locations is transboundary pollution which is modelled as a PDE diffusion equation. The spatio-temporal functional is quadratic in local consumption and linear in pollution. Using a dynamic programming method adapted to our infinite dimensional setting, we solve the associated optimal control problem in closed-form and identify the asymptotic (optimal) spatial distribution of pollution. We show that optimal emissions will decrease at given location if and only if local productivity is larger than a threshold which depends both on the local pollution absorption capacity and environmental awareness. Furthermore, we numerically explore the relationship between the spatial optimal distributions of production and (asymptotic) pollution in order to uncover possible (geographic) environmental Kuznets curve cases.
\end{abstract}

Mathematics Subject Classification. 49J20, 35K10, 35Q93.

Received May 3, 2018. Accepted December 13, 2018.

\section{INTRODUCTION}

A key question addressed in both economic and ecological research is the possibility to generate virtuous scenarios where economic development (say, increase in living standards) is associated with a non-catastrophic pattern in pollution. Some argue that such a possibility is remote and is not even desirable: degrowth proponents typically argue that sustainable development as for example understood as a path with a viable level of pollution cannot be managed if there is no significant cut in our standards of living (see e.g., [17], in the ecological economics literature, and [1], for a more neutral assessment of the above mentioned tradeoff).

Economic growth theorists and practitioners have sketched the problem into a different and to a certain extent more attractive formulation: Could it be the case that the stock of pollution, while initially rising, gets eventually curbed in posterior development stages? This pattern is now widely known as the Environmental Kuznets Curve (EKC hereafter). The idea behind such a conjecture is the following: in the initial stages of development, the

Keywords and phrases: Growth, geography, transboundary pollution, infinite dimensional optimal control problems.

1 Aix-Marseille University (Aix-Marseille School of Economics and IMéRA), CNRS, EHESS and Ecole Centrale de Marseille, Senior Member of the Institut Universitaire de France, Marseille, France.

${ }^{2}$ Univ. Grenoble Alpes, CNRS, INRA, Grenoble INP, GAEL, 38000 Grenoble, France.

3 Università degli Studi di Siena, Dipartimento di Economia Politica e Statistica, Siena, Italy.

4 Dipartimento di Economia e Finanza, LUISS Guido Carli, Rome, Italy.

* Corresponding author: giorgio.fabbri@univ-grenoble-alpes.fr 
economies are poor, technologically far lagged and the population is near starvation, increasing production is an absolute priority, and this normally goes with an increase in pollution; as the economies develop and both the living and technological standards go up, there is room for pushing environmental norms without hurting too much welfare. In other words, above a certain level of economic and technological development, it might well be possible to reduce pollution without affecting significantly production and welfare. Several theoretical papers have been written in this vein. A seminal work in this respect is due to Stokey [25]: within the framework of optimal growth, the author studied the possible occurrence of EKC depending on the objective functionals and the production technologies. It turns out that within such a canonical framework, EKC is by no way granted, it strongly depends on the shape of the production functions and the parameterization of the objective functionals (in economics, this mainly concerns the preferences of the social planner). The picture becomes overwhelmingly complicated if one departs from the canonical models developed in Stokey. For example, considering irreversible pollution (that's the case where above a certain level of pollution, nature regeneration capacity drops permanently) increases dramatically the set of possible (optimal) relationships between pollution and, say, production. See Prieur [21] and Boucekkine et al. [9].

On the empirical side, and as detailed in Stern [24], the literature is even more inconclusive irrespective of the data type used (cross country, time series for a fixed country or panel). A key finding is that the relationship between pollution and income greatly depends on the type of pollutant. Indeed, while sulfur dioxide and nitrous oxide emissions show an inverse $\mathrm{U}$ relationship with respect to income, $\mathrm{CO}_{2}$ seems to exhibit an $\mathrm{N}$ shape, not at all the EKC pattern (see [23]). These inconclusive reports have led several authors (see e.g., [5]) to argue that whenever it is observed, the EKC seems to be only a transitory phenomenon, at least for carbon emissions.

In this paper, we propose to retake the theoretical analysis avenue to especially dig deeper into the spatial determinants of the relationship between pollution and production (or income). Indeed, one very important characteristic of pollution (and in particular 5) is that it has a very significant transboundary component. While some of the related empirical work conducted takes into account the geographic variability, a very vast majority of the counterpart theoretical literature does not, not speaking about transboundary pollution. Only very recently, a few attempts to account for the latter can be noticed. Two articles are worth mentioning here. In first place, Camacho and Perez Barahona [12] have produced the first meaningful attempt to incorporate transboundary pollution (through a diffusion equation, see below) in a theoretical spatio-temporal model of optimal land use dynamics and environment-friendly abatement activities. Beside technicalities, the authors properly show how such a spatio-temporal setting is able to reproduce a large set of spatial patterns due to the interplay between land use and abatement activities together with pollution diffusion across space. La Torre et al. [? ] is another original spatio-temporal (linear-quadratic) model with transboundary pollution diffusion, specifically more concerned with local versus global environment-friendly policies (here, local versus global environmental taxes). The authors neatly show that local policies are essential to achieve the global environmental objectives.

Beside tackling a clearly different research question, we sharply depart from the two latter papers in terms of mathematical methodology. While these works use an adapted method of calculus of variations to infer the associated Pontryagin conditions, we resort to dynamic programming. Because the involved diffusion state equations are PDEs instead of ODEs, an appropriate method has to be employed to tackle the original optimal control problem. To this end, we build on Bensoussan et al. [6] (see also, for a recent related application in the spatio-temporal dynamics field, Boucekkine et al. [8]) rewriting, by a semigroup approach, the controlled PDE state equation as a controlled ODE in a suitable infinite dimensional (Hilbert) space. Then we exploit the linear-quadratic structure of the problem and the tools of infinite dimensional optimal control theory to explicitly compute the value function, the optimal control/state trajectories and the long-run optimal state. The results are then rewritten in the original PDE framework.

On the economic modelling side, we build on Boucekkine et al. [10]. In the original framework, two countries, affected by the same pollution (either, say, border water or green space polluted by the emissions of both, or mere $\mathrm{CO}_{2}$ transboundary pollution), have to decide in a non-cooperative way (Nash or Stackelberg) whether they have to switch to a cleaner but less efficient technology and when they have to do so. Importantly enough, transboundary pollution is not essential in this story. Indeed, the law of motion of pollution, shared by the 
two countries, is an ODE involving the emissions of both countries. Here, we move from a two-country to a continuous space setting, and transboundary pollution is accordingly modeled as a diffusion (PDE) process. Moreover, contrary to Camacho and Perez Barahona [12] or La Torre et al. [? ], time horizon is taken infinite, which allows us to inquire about asymptotic properties. We also remove the strategic aspects inherent in the original model of Boucekkine et al. [10] to concentrate on the implications of the geographic modelling. Indeed, we do enrich the model with several further geographical ingredients: we specifically assume that technology, nature self-cleaning capacity and public environmental awareness are space dependent. While the first specification is a common assumption in the related literature (see again [12], or Boucekkine et al. [8]), the two latter ones are not though they are quite natural. In particular, it seems most realistic to assume that people living in different places (countries or regions) may not share the same reluctance to pollution and the same awareness regarding the impact of environmental deterioration, we model this by introducing a space-dependent parameter in the functional to reflect differences in pollution perception across space (see [22], for a pioneering non-geographic theoretical approach).

Thanks to the linear quadratic framework, we are able to generate closed-form solutions, which in turn allow us to have an accurate view of how the geographic ingredients may affect the relationship between pollution and production. We concentrate on the relationship at the optimum between the spatial distribution of production and the (asymptotic) spatial distribution of pollution. In this sense, we investigate to which geographic EKC may arise in our canonical framework and under which conditions. The rest of the paper is structured as follows. Section 2 presents the general associated problem setting and provides with all the steps of the applied dynamic programming method. General closed-form solutions for optimal pollution and production patterns are provided. Section 3 is twofold. It first presents the geographic and non-strategic version of Boucekkine et al. [10] and establishes the link with the general setting. Next, we deliver some preliminary theoretical results on how the spatial distribution of optimal input uses and emissions is affected by the spatial distributions of technology, nature self-cleaning capacity and awareness. A general condition involving the latter three distributions is derived for local productivity to curb local emissions. Not only technology (locally) matters: also ecology and culture do matter. In Section 4, the optimal patterns generated are explored numerically and exploiting the richness of the geographic heterogeneity factors. Section 5 concludes.

\section{THE MODEL}

As indicated in the introduction, the economic model is inspired from the dynamic game framework of Boucekkine et al. [10]. Instead of having two competing regions, we have a continuum of locations, say along $\mathbb{R}$, but no strategic interaction for simplification. Each location uses a Leontief (linear) production function (that's a technology which combines various inputs in fixed proportions, e.g. labor and capital). Consider an input which is a flow, this requirement comes from the need to work with only one state equation describing pollution spatio-temporal dynamics, a stock input would add another one and complicates the analysis. The production function at location $x$ in time $t \geq 0$ writes as:

$$
y(t, x)=A(x) i(t, x)
$$

where $y(t, x)$ is the output, $i(t, x)$ is the input, and $A(x)$ is productivity at location $x$. We assume that $A(\cdot)$ is time independent for simplification. ${ }^{1}$ At any location, output is produced, consumed and used as input locally, leading to:

$$
c(x, t)+i(t, x)=y(t, x)
$$

with obvious notations. Equation (2.2) implies that there is no trade in the good across regions. The unique link between the locations is transboundary pollution. Assume that $\left(\mathrm{CO}_{2}\right)$ emissions at location $x$ are exactly

\footnotetext{
${ }^{\mathrm{I}}$ We could have developed the general setting for exponentially growing productivity over time.
} 
equal to input use intensity, $i(t, x)$. The spatio-temporal dynamics of the stock of local pollution, $p(t, x)$, follows a diffusion equation in $\mathbb{R}$, that is:

$$
\left\{\begin{array}{l}
\frac{\partial p}{\partial t}(t, x)=\frac{\sigma^{2}}{2} \Delta p(t, x)-\delta(x) p(t, x)+i(t, x), \quad(t, x) \in \mathbb{R}^{+} \times \mathbb{R}, \\
p(0, x)=p_{0}(x), \quad x \in \mathbb{R} .
\end{array}\right.
$$

Pollution in location $x$ at time $t$ depends on transboundary pollution diffusion (the term $\frac{\sigma^{2}}{2} \Delta p(t, x)$ ), on nature local regeneration capacity (the term $\delta(x) p(t, x)$ ), and on current emissions (as captured by the term $i(t, x)$ ).

The objective functional will be an infinite horizon time-discounted integral (in time and space) of an instantaneous utility function which depends on local consumption and pollution. To get closed-form solutions, we assume that the instantaneous utility function per location, $U(c, p)$, is quadratic in consumption and linear in the pollution stock. More precisely, we assume that:

$$
U(c(t, x), p(t, x))=c(t, x)-c^{2}(t, x)-w(x) p(t, x),
$$

where $w(x)$ measures environmental awareness in location $x$. Using $(2.1)-(2.2)$ to substitute $c(t, x)$ for $i(t, x)$, one gets an intertemporal functional of the form:

$$
\int_{0}^{\infty} e^{-\rho t}\left(\int_{\mathbb{R}}\left(a(x) i(t, x)-\frac{1}{2} b(x) i(t, x)^{2}-w(x) p(t, x)\right) \mathrm{d} x\right) \mathrm{d} t
$$

with

$$
a(x)=A(x)-1,
$$

and

$$
b(x)=2 a^{2}(x) .
$$

Accordingly, we may also interpret $a(x)$ as productivity at location $x$. These restrictions will be useful below to solve the problem and to approximate numerically the solutions.

\section{The GENERAL MATHEMATiCAL PROBLEM AND ITS SOLUTiON}

\subsection{The setting}

We consider the following optimal control problem in $\mathbb{R}^{n}, n \geq 1$,

$$
\left\{\begin{array}{l}
\frac{\partial p}{\partial t}(t, x)=\frac{\sigma^{2}}{2} \Delta p(t, x)-\delta(x) p(t, x)+i(t, x), \quad(t, x) \in \mathbb{R}^{+} \times \mathbb{R}^{n}, \\
p(0, x)=p_{0}(x), \quad x \in \mathbb{R}^{n},
\end{array}\right.
$$

where $p_{0}, \delta: \mathbb{R}^{n} \rightarrow \mathbb{R}$ are given measurable functions, $\sigma>0, \Delta$ is the Laplacian operator in $\mathbb{R}^{n}$, i.e.

$$
\Delta \varphi=\sum_{k=1}^{n} \frac{\partial^{2} \varphi}{\partial x_{k}^{2}}, \quad x=\left(x_{1}, \ldots, x_{n}\right), \quad \varphi \in C^{2}\left(\mathbb{R}^{n}\right),
$$

and $i: \mathbb{R}^{+} \times \mathbb{R}^{n} \rightarrow \mathbb{R}$ is the control function.

Since we take the problem where the space variable varies in the whole space $\mathbb{R}^{n}$ the boundary conditions should be formulated as limiting condition when $\|x\| \rightarrow+\infty$. Such a condition is hidden here in the fact that, 
as we see below at the beginning of Section 3.2 , we embed the problem in the space $L^{2}\left(\mathbb{R}^{n}\right)$, which already includes a limiting condition.

The goal is to maximize the functional

$$
\int_{0}^{\infty} e^{-\rho t}\left(\int_{\mathbb{R}^{n}}\left(a(x) i(t, x)-\frac{1}{2} b(x) i(t, x)^{2}-w(x) p(t, x)\right) \mathrm{d} x\right) \mathrm{d} t
$$

where $\rho>0$, where $a, b, w: \mathbb{R}^{n} \rightarrow \mathbb{R}$ are given functions and $y$ in the integral above is the solution (in a sense to be clarified) to (3.1) corresponding to the initial datum $p_{0}$ and to the control $i$ belonging to a suitable class of admissible controls.

\subsection{Hilbert space formulation}

Consider the space

$$
L^{2}\left(\mathbb{R}^{n}\right):=\left\{f: \mathbb{R}^{n} \rightarrow \mathbb{R} \text { measurable }: \int_{\mathbb{R}^{n}}|f(x)|^{2} \mathrm{~d} x<\infty\right\}
$$

endowed with the with inner product

$$
\langle f, g\rangle:=\int_{\mathbb{R}^{n}} f(x) g(x) \mathrm{d} x, \quad f, g \in L^{2}\left(\mathbb{R}^{n}\right),
$$

which renders it a Hilbert space. ${ }^{2}$ Denote by $\|\cdot\|$ the associated norm, i.e.

$$
\|f\|^{2}:=\int_{\mathbb{R}^{n}}|f(x)|^{2} \mathrm{~d} x, \quad f \in L^{2}\left(\mathbb{R}^{n}\right)
$$

We introduce the following assumptions, which will be standing in the rest of the section. ${ }^{3}$

\section{Assumption 3.1.}

(i) $p_{0}, a, w \in L^{2}\left(\mathbb{R}^{n}\right)$ and $p_{0}, w$ are nonnegative;

(ii) $\delta, b: \mathbb{R}^{n} \rightarrow \mathbb{R}$ are bounded and $b \geq b_{0}, \delta \geq \delta_{0}$ for some $b_{0}, \delta_{0}>0$.

Set $H:=L^{2}\left(\mathbb{R}^{n}\right)$. We rigorously reformulate the control problem described above in the space $H$ as follows. Denote by $L(H)$ the space of bounded linear operators on $H$. Consider the diffusion semigroup $\{T(t)\}_{t \geq 0} \subset L(H)$ defined by

$$
[T(t) \varphi](x):=\frac{1}{\sqrt{4 \pi} t^{n / 2}} \int_{\mathbb{R}^{n}} e^{-\frac{|\xi|^{2}}{4 t}} \varphi(x+\xi) \mathrm{d} \xi, \quad \varphi \in H
$$

\footnotetext{
${ }^{2}$ To be precise, the space $L^{2}\left(\mathbb{R}^{n}\right)$ is done by equivalence classes of functions according to the equivalence relation which identifies functions which are equal almost everywhere (see [?]).

${ }^{3}$ In the case we are interested in Section 2 (see also Sects. 4 and 5), taking the coefficient $A$ bounded and the coefficient $b(x)=2(A(x)-1))^{2}$ satisfying the conditions of part (ii) of Assumption 3.1 would imply that $\left.a(x)=(A(x)-1)\right)$ cannot directly satisfy part (i) of Assumption 3.1. Nevertheless, we can consider a "localization" of $a(\cdot)$ by considering $\tilde{a}_{R}(x)=\psi_{R}(x) a(x)$, where $\psi_{R} \equiv 1$ on $[-R, R]$ for some fixed "big" $R>0$ and is rapidly decreasing to 0 for $|x|>R$. Then we have $a(x)=\lim _{R \rightarrow+\infty} \tilde{a}_{R}(x)$ for all $x \in \mathbb{R}$. The functions $\tilde{a}$ and $b$ defined in this way satisfy Assumption 3.1. Moreover, given any compac set $[-M, M] \subset \mathbb{R}$, the corresponding optimal control $i_{R}^{*}$ given in (3.18) is stable as $R \rightarrow \infty$, meaning that it does not depend on $R$ and on the choice of $\psi_{R}$ as soon as $R_{M}$. The expression of $i^{*}$ that we use in (4.1) is then the natural one consisting in considering $i_{R}^{*}$ at each point when $R \rightarrow \infty$ in the procedure above.
} 
It is well known (see [15], pp. 69-70) that $\{T(t)\}_{t \geq 0}$ is a strongly continuous semigroup of operators in $L(H)$. Its infinitesimal generator (see [20], p. 72 , Sect. 3.1.1) is $(\mathcal{A}, D(\mathcal{A}))$ where

$$
D(\mathcal{A})=W^{2,2}\left(\mathbb{R}^{n}\right), \quad \mathcal{A} \varphi=\Delta \varphi, \quad \forall \varphi \in D(\mathcal{A}) .
$$

A core for the latter is the Schwartz space $\mathcal{S}\left(\mathbb{R}^{n}\right)$ (see again [15], pp.69-70). A double integration by parts shows that

$$
\langle\mathcal{A} \varphi, \psi\rangle=\langle\varphi, \mathcal{A} \psi\rangle, \quad\langle\mathcal{A} \varphi, \varphi\rangle \leq 0, \quad \forall \varphi, \psi \in \mathcal{S}\left(\mathbb{R}^{n}\right)
$$

Since $\mathcal{S}\left(\mathbb{R}^{n}\right)$ is core for $\mathcal{A}$, the latter extend to $D(\mathcal{A})$ showing that $\mathcal{A}$ is self-adjoint and dissipative. Next, consider the multiplicative operators in $L(H)$

$$
[\mathcal{D} \varphi](x)=-\delta(x) \varphi(x), \quad[\mathcal{B} \varphi](x)=b(x) \varphi(x),
$$

and set

$$
\mathcal{L}:=\frac{\sigma^{2}}{2} \mathcal{A}+\mathcal{D}, \quad D(\mathcal{L})=D(\mathcal{A})
$$

$\mathcal{D}$ is clearly a self-adjoint and dissipative operator of $L(H)$. So, also $\mathcal{L}$ is self-adjoint and dissipative.

Given this framework, with the formal equalities $P(t)(x)=p(t, x)$ and $I(t)(x)=i(t, x)$, we naturally reformulate (3.1) in $H$ as

$$
\left\{\begin{array}{l}
P^{\prime}(t)=\mathcal{L} P(t)+I(t), \quad t \geq 0 \\
P(0)=p_{0} \in H
\end{array}\right.
$$

where $I$ belongs to the set of admissible controls

$$
\mathcal{I}:=\left\{I: \mathbb{R}^{+} \rightarrow H \text { measurable }: \int_{0}^{\infty} e^{-\rho t}\|I(t)\|^{2} \mathrm{~d} t<\infty\right\}
$$

According to ([6], Part. II, Chap. 1. Def. 3.1(v)), given $p_{0} \in H$ and $I \in \mathcal{I}$, we define the mild solution to (3.3) as

$$
P\left(t ; p_{0}, I\right):=e^{t \mathcal{L}} p_{0}+\int_{0}^{t} e^{(t-s) \mathcal{L}} I(s) \mathrm{d} s, \quad t \geq 0 .
$$

The functional (3.2) is then rewritten in this abstract framework as

$$
J\left(p_{0} ; I\right):=\int_{0}^{+\infty} e^{-\rho t}\left(\langle a, I(t)\rangle-\frac{1}{2}\langle\mathcal{B} I(t), I(t)\rangle-\left\langle w, P\left(t ; p_{0}, I\right)\right\rangle\right) \mathrm{d} t .
$$

\subsection{Solution by dynamic programming}

We define the value function of the problem

$$
v\left(p_{0}\right):=\sup _{I \in \mathcal{I}} J\left(p_{0}, I\right)
$$


The Hamilton-Jacobi-Bellman (HJB) equation associated to $v$ is formally written as

$$
\rho v\left(p_{0}\right)=\left\langle\mathcal{L} p_{0}, \nabla v\left(p_{0}\right)\right\rangle-\left\langle w, p_{0}\right\rangle+\sup _{I_{0} \in H}\left\{\left\langle I_{0}, \nabla v\left(p_{0}\right)\right\rangle+\left\langle a, I_{0}\right\rangle-\frac{1}{2}\left\langle\mathcal{B} I_{0}, I_{0}\right\rangle\right\}
$$

Taking into account that $\mathcal{L}=\mathcal{L}^{*}$ we rewrite HJB (3.6) as

$$
\rho v\left(p_{0}\right)=\left\langle p_{0}, \mathcal{L} \nabla v\left(p_{0}\right)\right\rangle-\left\langle w, p_{0}\right\rangle+\sup _{I_{0} \in H}\left\{\left\langle I_{0}, \nabla v\left(p_{0}\right)\right\rangle+\left\langle a, I_{0}\right\rangle-\frac{1}{2}\left\langle\mathcal{B} I_{0}, I_{0}\right\rangle\right\}
$$

We look for a solution in the form

$$
v\left(p_{0}\right)=\left\langle\alpha, p_{0}\right\rangle+\beta_{0}
$$

where $\alpha \in D(\mathcal{L}), \beta_{0} \in \mathbb{R}$. With this structure we have

$$
\sup _{I_{0} \in H}\left\{\left\langle I_{0}, \nabla v\left(p_{0}\right)\right\rangle+\left\langle a, I_{0}\right\rangle-\frac{1}{2}\left\langle\mathcal{B} I_{0}, I_{0}\right\rangle\right\}=\frac{1}{2}\left\langle a+\alpha, \mathcal{B}^{-1}(a+\alpha)\right\rangle
$$

with optimizer

$$
I_{0}^{*}=\mathcal{B}^{-1}(a+\alpha)
$$

noticing that $\mathcal{B}^{-1} \in L(H)$ due to Assumption 3.1; precisely:

$$
\left[\mathcal{B}^{-1} \varphi\right](x)=\frac{\varphi(x)}{b(x)}, \quad \varphi \in H
$$

Note that $I_{0}^{*}$ does not depend on $p_{0}$, as typically happens in linear-quadratic control. Hence, given (3.8) and (3.9), HJB (3.7) becomes

$$
\rho\left(\left\langle\alpha, p_{0}\right\rangle+\beta_{0}\right)=\left\langle p_{0}, \mathcal{L} \alpha\right\rangle-\left\langle w, p_{0}\right\rangle+\frac{1}{2}\left\langle a+\alpha, \mathcal{B}^{-1}(a+\alpha)\right\rangle
$$

Since $\mathcal{L}$ is dissipative and $\rho>0$, it follows (see [15], Prop. 3.14, p. 82) that $\rho$ belongs to the resolvent set of $\mathcal{L}$, i.e.

$$
\rho-\mathcal{L}: D(\mathcal{L}) \longrightarrow H
$$

is invertible with bounded inverse $(\rho-\mathcal{L})^{-1}: H \rightarrow H$. Equating the terms containing $p_{0}$ and the terms not containing it, we see that (3.8) is a solution to (3.11) if and only if

$$
\alpha=-(\rho-\mathcal{L})^{-1} w \in D(\mathcal{L}), \quad \beta_{0}=\frac{1}{2 \rho}\left\langle a+\alpha, \mathcal{B}^{-1}(a+\alpha)\right\rangle
$$


Since $I_{0}^{*}$ does not depend on $p_{0}$, the formal optimal control ${ }^{4} I^{*} \in \mathcal{I}$ is constant, i.e.

$$
I^{*}(t) \equiv I_{0}^{*}=\mathcal{B}^{-1}(a+\alpha),
$$

and the closed loop equation associated to it is actually just a linear non-homogeneous differential equation in $H$ :

$$
\left\{\begin{array}{l}
P^{\prime}(t)=\mathcal{L} P(t)+\mathcal{B}^{-1}(a+\alpha), \\
P(0)=p_{0} .
\end{array}\right.
$$

Its solution is

$$
P^{*}\left(t ; p_{0}\right)=e^{t \mathcal{L}} p_{0}+\int_{0}^{t} e^{(t-s)} \mathcal{L}_{\mathcal{B}^{-1}}(a+\alpha) \mathrm{d} s .
$$

The limit distribution as $t \rightarrow \infty$ can be obtained by splitting

$$
\mathcal{L}=\mathcal{L}_{0}+\mathcal{D}_{0},
$$

where

$$
\mathcal{L}_{0}:=\frac{\sigma^{2}}{2} \mathcal{A}+\left(\mathcal{D}-\mathcal{D}_{0}\right)
$$

and

$$
\mathcal{D}_{0} \varphi:=-\delta_{0} \varphi, \quad \varphi \in H
$$

where $\delta_{0}$ is the constant of Assumption 3.1. Indeed, we can rewrite

$$
P^{*}(t)=e^{-\delta_{0} t} e^{t \mathcal{L}_{0}} p_{0}+\int_{0}^{t} e^{-\delta_{0}(t-s)} e^{(t-s) \mathcal{L}_{0}} I^{*} \mathrm{~d} s,
$$

and take the limit above when $t \rightarrow \infty$. Noticing that $\mathcal{L}_{0}$ is dissipative too, hence $e^{s \mathcal{L}_{0}}$ is a contraction, the first term of the right hand side converges to 0 , whereas the second one converges to

$$
P_{\infty}^{*}:=\int_{0}^{\infty} e^{-\delta_{0} s} e^{s \mathcal{L}_{0}} I^{*} \mathrm{~d} s
$$

By standard theory of strongly continuous semigroups ([15], Chap. II, Thm.1.10), we have

$$
P_{\infty}^{*}=\left(\delta_{0}-\mathcal{L}_{0}\right)^{-1} I^{*}
$$

\footnotetext{
${ }^{4} \mathrm{~A}$ verification theorem can be proved (see, e.g., in a similar context, [16]) to prove that $v$ defined by (3.8) with the specifications given in (3.12) is really the value function and that (3.14) is really an optimal control. We omit the proof for brevity. It relies on the fact that (3.8) solves HJB (3.6) and that, by ([6], Part. II, Chap. 1. Prop. 3.2), the mild solution to (3.3) is also a weak solution (actually the unique) to the same equation, i.e., taking into account that $\mathcal{L}$ is self-adjoint, i.e. $\mathcal{L}=\mathcal{L}^{*}$, and setting $P(t):=P\left(t ; p_{0}, I\right)$, it holds
}

$$
\langle P(t), \varphi\rangle=\left\langle p_{0}, \varphi\right\rangle+\int_{0}^{t}(\langle P(s), \mathcal{L} \varphi\rangle+\langle I(s), \varphi\rangle) \mathrm{d} s \quad \forall t \geq 0, \quad \forall \varphi \in D(\mathcal{L})
$$


Summarizing and translating the results in the form needed for the original control problem (3.1)-(3.2), we get the following.

\section{Proposition 3.2.}

(i) The value function of the control problem is

$$
v\left(p_{0}\right)=\int_{\mathbb{R}^{n}} \alpha(x) p_{0}(x) \mathrm{d} x+\beta_{0}, \quad \forall p_{0} \in H
$$

where $\alpha$ is the unique solution in $H$ to the elliptic PDE

$$
\rho \alpha(x)-\frac{\sigma^{2}}{2} \Delta \alpha(x)+\delta(x) \alpha(x)=-w(x)
$$

and $\beta_{0}$ is the constant given by

$$
\beta_{0}=\frac{1}{2 \rho} \int_{\mathbb{R}^{n}} \frac{(a(x)+\alpha(x))^{2}}{b(x)} \mathrm{d} x .
$$

(ii) The optimal control $i^{*}$ is given by

$$
i^{*}(t, x):=I^{*}(t)(x)=\frac{a(x)+\alpha(x)}{b(x)}, \quad(t, x) \in \mathbb{R}^{+} \times \mathbb{R}^{n},
$$

constant in time, not in space.

(iii) The optimal state $p^{*}(t, x):=P^{*}\left(t ; p_{0}\right)(x)$ is the solution to

$$
\left\{\begin{array}{l}
\frac{\partial p(t, x)}{\partial t}=\frac{\sigma^{2}}{2} \Delta p(t, x)-\delta(x) p(t, x)+\frac{a(x)+\alpha(x)}{b(x)}, \quad(t, x) \in \mathbb{R}^{+} \times \mathbb{R}^{n}, \\
p(0, x)=p_{0}(x), \quad x \in \mathbb{R}^{n} .
\end{array}\right.
$$

(iv) The limit distribution $p_{\infty}^{*}(x):=\lim _{t \rightarrow \infty} p^{*}(t, x)$ is the unique solution to the elliptic PDE

$$
\delta(x) p_{\infty}^{*}(x)-\frac{\sigma^{2}}{2} \Delta p_{\infty}^{*}(x)=I^{*}(x),
$$

Proof. By (3.12), we have $(\rho-\mathcal{L}) \alpha=-w$, which is the abstract version of (3.17), proving (i). Similarly (3.15) is the abstract version of (3.19) and (3.16) is the abstract version of (3.20), proving the other claims.

We notice that the solution $\alpha$ to (3.17) in $H$ admits the probabilistic representation (see [18], Exercise 4.7, Sect. 4.4.A)

$$
\alpha(x)=-\mathbb{E}\left[\int_{0}^{\infty} e^{-\left(\rho t+\int_{0}^{t} \delta\left(x+\sigma W_{s}\right) \mathrm{d} s\right)} w\left(x+\sigma W_{t}\right) \mathrm{d} t\right]
$$

where $W$ is a standard $n$-dimensional Brownian motion. Moreover, if $\delta(x) \equiv \delta_{0} \geq 0$ is constant, it admits the integral representation

$$
\alpha(x)=-\int_{0}^{\infty} e^{-\left(\rho+\delta_{0}\right) t}\left(\int_{\mathbb{R}^{n}} \frac{1}{\sqrt{2 \pi\left(\sigma^{2} t\right)^{n}}} e^{-\frac{|\xi|^{2}}{2 \sigma^{2} t}} w(x+\xi) \mathrm{d} \xi\right) \mathrm{d} t .
$$


Similarly, setting

$$
f(x):=\frac{a(x)+\alpha(x)}{b(x)}, \quad x \in \mathbb{R}^{n}
$$

$p^{*}$ admits the probabilistic representation (see [18], Thm. 4.2, Sect.4.4.A)

$$
p^{*}(t, x)=\mathbb{E}\left[p_{0}\left(x+\sigma W_{t}\right) e^{-\int_{0}^{t} \delta\left(x+\sigma W_{r}\right) \mathrm{d} r}+\int_{0}^{t} e^{-\int_{0}^{s} \delta\left(x+\sigma W_{r}\right) \mathrm{d} r} f\left(x+\sigma W_{s}\right) \mathrm{d} s\right]
$$

where $W$ is a standard $n$-dimensional Brownian motion, and, if $\delta(x) \equiv \delta_{0}>0$ is constant, then the latter admits the integral representation

$$
\begin{aligned}
p^{*}(t, x)= & e^{-\delta_{0} t} \int_{\mathbb{R}^{n}} \frac{1}{\sqrt{2 \pi\left(\sigma^{2} t\right)^{n}}} e^{-\frac{|\xi|^{2}}{2 \sigma^{2} t}} p_{0}(x+\xi) \mathrm{d} \xi \\
& +\int_{0}^{t} e^{-\delta_{0} s}\left(\int_{\mathbb{R}^{n}} \frac{1}{\sqrt{2 \pi\left(\sigma^{2} s\right)^{n}}} e^{-\frac{|\xi|^{2}}{2 \sigma^{2} s}} f(x+\xi) \mathrm{d} \xi\right) \mathrm{d} s
\end{aligned}
$$

Finally, for the same reason as before, $p_{\infty}^{*}$ has the probabilistic representation:

$$
p_{\infty}^{*}(x)=\mathbb{E}\left[\int_{0}^{\infty} e^{-\int_{0}^{s} \delta\left(x+\sigma W_{r}\right) \mathrm{d} r} i^{*}\left(x+\sigma W_{s}\right) \mathrm{d} s\right]
$$

where $W$ is a standard $n$-dimensional Brownian motion. If $\delta(x) \equiv \delta_{0}>0$ is constant, then the latter admits the integral representation

$$
p_{\infty}^{*}(x)=\int_{0}^{\infty} e^{-\delta_{0} t}\left(\int_{\mathbb{R}^{n}} \frac{1}{\sqrt{2 \pi\left(\sigma^{2} t\right)^{n}}} e^{-\frac{|\xi|^{2}}{2 \sigma^{2} t}} i^{*}(x+\xi) \mathrm{d} \xi\right) \mathrm{d} t .
$$

\section{Remark 3.3.}

(i) We observe that a technique similar to the one used here to solve the problem (i.e. guessing an affine form of the value function and using it to represent to optimal strategies and trajectories) has already been used in other papers, like $[2-4,16]$, in the case when the state equation is a first order PDE (not a second order one like here) and the space variable belongs to a subset of $\mathbb{R}_{+}$. Hence the results presented here can be seen as a new application of such approach to problems with second order state equation and, possibly, multidimensional in space.

(ii) We think it may be possible to generalize the above results to the case when the utility is not a linear quadratic one. However, the linearity of the state equation and the fact that the objective functional depends linearly on the state are needed here to use our method of solution.

(iii) It is important to note that, see (3.18), the optimal investment here is constant in time. This comes from the fact that the state variable $p$ appears linearly both in the state equation and in the objective functional. This is different, for example, from the models of $[7,8]$, where the state equation is linear, but the objective functional (when $i$ is taken as control) does not depend linearly in the state variable. Nevertheless, also in the present model the pollution has a transitional dynamics that we do not investigate here, limiting ourselves to the study of the limit distribution.

(iv) It may be possible to write our model taking a discrete space variable (see e.g. [13]). The continuous space model allows to treat the problem by analytical techniques and to find the explicit solutions of the problem. 
(v) In our model we have a situation of full capital depreciation. Eliminating this assumption would require to explicitly add in our model, beyond the law of motion for the pollution, also the law of motion of capital, which could be written, e.g., following $[7,8]$. This would be an interesting extension of our model that we aim to address in future works.

\section{TheOretical RESUlts ON THE Optimal SPATIAL DisTRIBUtion OF EMISSIONS}

Before, a prior quick analytical perspective is worth doing. One important thing to notice is that optimal input use, and therefore emissions, are constant per location:

$$
i^{*}(t, x)=i^{*}(x)=\frac{a(x)+\alpha(x)}{b(x)}, \quad(t, x) \in \mathbb{R}^{+} \times \mathbb{R} .
$$

Because optimal investment is time-independent (due to the linear quadratic setting) and because the problem is autonomous (i.e productivity, nature self-cleaning capacity and environmental awareness functions are time independent), optimal production is also time-independent. This is the perfect framework to concentrate on geography, and indeed we shall explore the relationship between the spatial optimal distributions of production and (asymptotic) pollution as explained later.

Suppose $\delta(x) \equiv \delta_{0} \geq 0$ and $w(x) \equiv w_{0}$ to fix the ideas. Then, as one can infer from the closed-form of $\alpha(x)$ in Section $2, \alpha(x)$ is constant across locations, that's

$$
\alpha(x) \equiv-\alpha_{0},
$$

with $\alpha_{0}$ is a well defined positive constant. With the restrictions implied by the economic model, optimal input use or the flow of emissions becomes

$$
i^{*}(x)=\frac{a(x)-\alpha_{0}}{2 a^{2}(x)}, \quad(t, x) \in \mathbb{R}^{+} \times \mathbb{R}
$$

with $a(x)=A(x)-1$. This immediately yields the following economically interesting proposition.

Proposition 4.1. Suppose $\delta(x) \equiv \delta_{0} \geq 0$ and $w(x) \equiv w_{0}$ (implying $\left.\alpha(x) \equiv-\alpha_{0}\right)$. Emissions $i^{*}(x)$ are a nonincreasing function of productivity $a(x)$ if and only if $a(x) \geq 2 \alpha_{0}$.

This is a quite nice property of the model regarding the EKC problem. As argued in the introduction (see also [?], for a more extensive survey), one of the main arguments behind the EKC is that as the economies develop and get more efficient (higher $a$ in our case), the more likely pollution get curbed along with development. The fact that optimal emissions are decreasing if and only if the locations are technologically enough in advance is a good indication that a geographic $\mathrm{EKC}$ (i.e the most advanced the location in terms of technology, $A(x)$, and/or production, $y^{*}(x)=A(x) i^{*}(x)=(a(x)+1) i^{*}(x)$, the lower the stock of pollution at $\left.x\right)$ may well hold. One way to pose the geographic EKC problem is to look at the correlation between production $y^{*}(x)$ and asymptotic pollution, $p_{\infty}^{*}(x)$. If $\delta(x) \equiv \delta_{0} \geq 0$ is constant, we know from Section 2 that the latter admits the following integral representation

$$
p_{\infty}^{*}(x)=\int_{0}^{\infty} e^{-\delta_{0} t}\left(\int_{\mathbb{R}} \frac{1}{\sqrt{2 \pi \sigma^{2} t}} e^{-\frac{|\xi|^{2}}{2 \sigma^{2} t}} i^{*}(x+\xi) \mathrm{d} \xi\right) \mathrm{d} t .
$$

We shall investigate the comovements between $y^{*}(x)$ and $p_{\infty}^{*}(x)$ numerically in the next section knowing that the above stated property leaves room for geographic EKC to emerge at the optimum. 

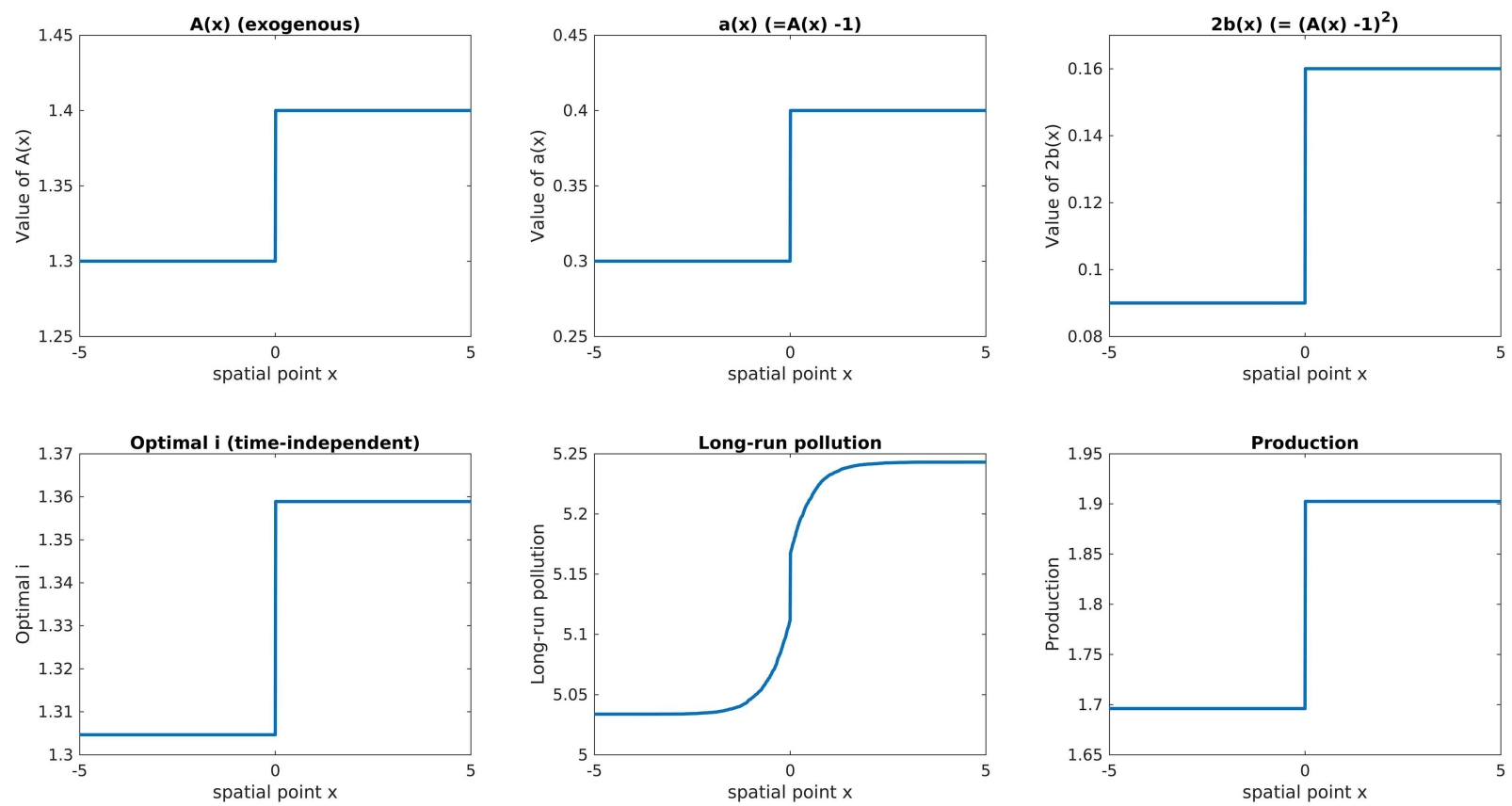

Figure 1. Optimal input profile, long-run pollution and production given the specified 2-steps distribution of the productivity $A(\cdot)$ (low $A$ case). Environmental awareness $w(\cdot)$ is here spaceindependent and equal to 0.05 , the self-cleaning capacity $\delta(\cdot)$ is space-independent and equal to $0.3, \rho=0.02, \sigma=\sqrt{2}$.

Let's now get to the case where neither self-cleaning capacity nor awareness are space-independent, that's the general case. Then the following result can be trivially extracted.

Proposition 4.2. Take any functions $\delta(x)$ and $w(x)$ satisfying assumptions of Section 3 (and the resulting function $\alpha(x)$ as defined Sect. 2). Further define $\alpha_{0}(x)$ as $\alpha_{0}(x)=-\alpha(x)>0$. Emissions $i^{*}(x)$ are a nonincreasing function of productivity $a(x)$ if and only if $a(x) \geq 2 \alpha_{0}(x)$.

This proposition generalizes the former one by introducing ecology $(\delta(x))$ and culture and/or psychology $(w(x))$ into the analysis. Indeed since $\alpha(x)$ is given by

$$
\alpha(x)=-\mathbb{E}\left[\int_{0}^{\infty} e^{-\left(\rho t+\int_{0}^{t} \delta\left(x+\sigma W_{s}\right) \mathrm{d} s\right)} w\left(x+\sigma W_{t}\right) \mathrm{d} t,\right]
$$

not only technology matters, both ecology and culture matter locally too. These factors are absent in the standard EKC studies in economics (see e.g., [25]). Here, the economic-ecologico-cultural balance delivers the following outcome: optimal emissions will decrease at location $x$ if and only if productivity is larger than a threshold which depends both on the local pollution absorption capacity and environmental awareness. As before, we can proceed now to the numerical exploration of the relationship between the optimal spatial distributions of production and (asymptotic) pollution (see Sect. 2 for the formula of $p_{\infty}^{*}(x)$ in the general case). 

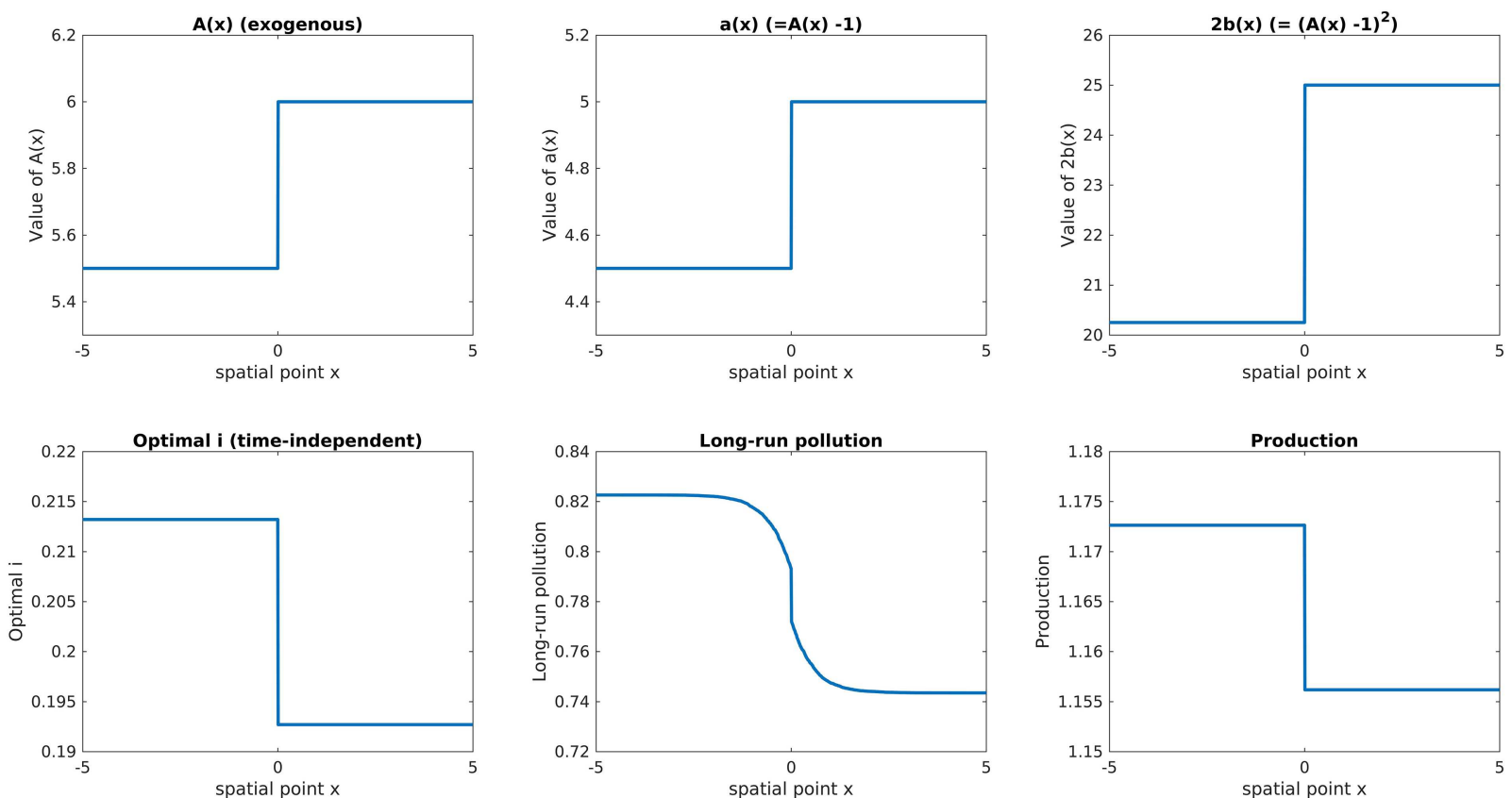

FIGURE 2. Optimal input profile, long-run pollution and production given the specified 2-steps distribution of the productivity $A(\cdot)$ (high $A$ case). Environmental awareness $w(\cdot)$ is here spaceindependent and equal to 0.05 , the self-cleaning capacity $\delta(\cdot)$ is space-independent and equal to $0.3, \rho=0.02, \sigma=\sqrt{2}$.

\section{Numerical EXPLORATION OF THE RELATIONSHIP BETWEEN THE SPATIAL OPTIMAL DISTRIBUTIONS OF POLLUTION AND PRODUCTION}

We shall consider in our numerical exercises very simple geographic functions for both productivity, environmental awareness and self-cleaning capacity, we choose piecewise constant functions to reflect North-South or North-Intermediate-South configurations. We could have also explored core-periphery settings but the conclusions are of course not altered. We proceed this way: we explore the implications of each geographic heterogeneity separately and then we put them together.

\subsection{The impact of geographic heterogeneity in productivity}

In the first two numerical exercises (Figs. 1 and 2) we look at the effect of a heterogeneous distribution of the productivity $A(\cdot)$. More precisely we consider a two-steps (North-South) spatial distribution of the productivity $A(\cdot)$ which is lower in the negative half-line (South) and higher in the positive half-line (North). As already emphasized in Propositions 4.1 and 4.2, the impact of increasing the productivity $A(\cdot)$ is ambiguous so that, depending on the general level of $A$, areas with higher productivity can use more (Fig. 1) or less (Fig. 2) input and are in the long run more or less polluted.

To emphasize the effect of geographic heterogeneity in productivity, in both the simulations we use a spacehomogeneous environmental awareness $w(\cdot)$ (whose value is equal to 0.05 in each point of space) and a spacehomogeneous self-cleaning capacity $\delta(\cdot)$ (equal to 0.3 in each spatial point). Other parameters are $\rho=0.02$ and $\sigma=\sqrt{2}$.

This ambiguous effect of the productivity on the long-run pollution is even more clearly accentuated in Figure 3 where we see what can happen in the context of a three-steps (North-Intermediate-South) productivity distribution. We have here a value of $A(\cdot)$ equal to 1.2 on the left of -2 , equal to 1.7 on $[-2,2]$ and to 2.2 on 

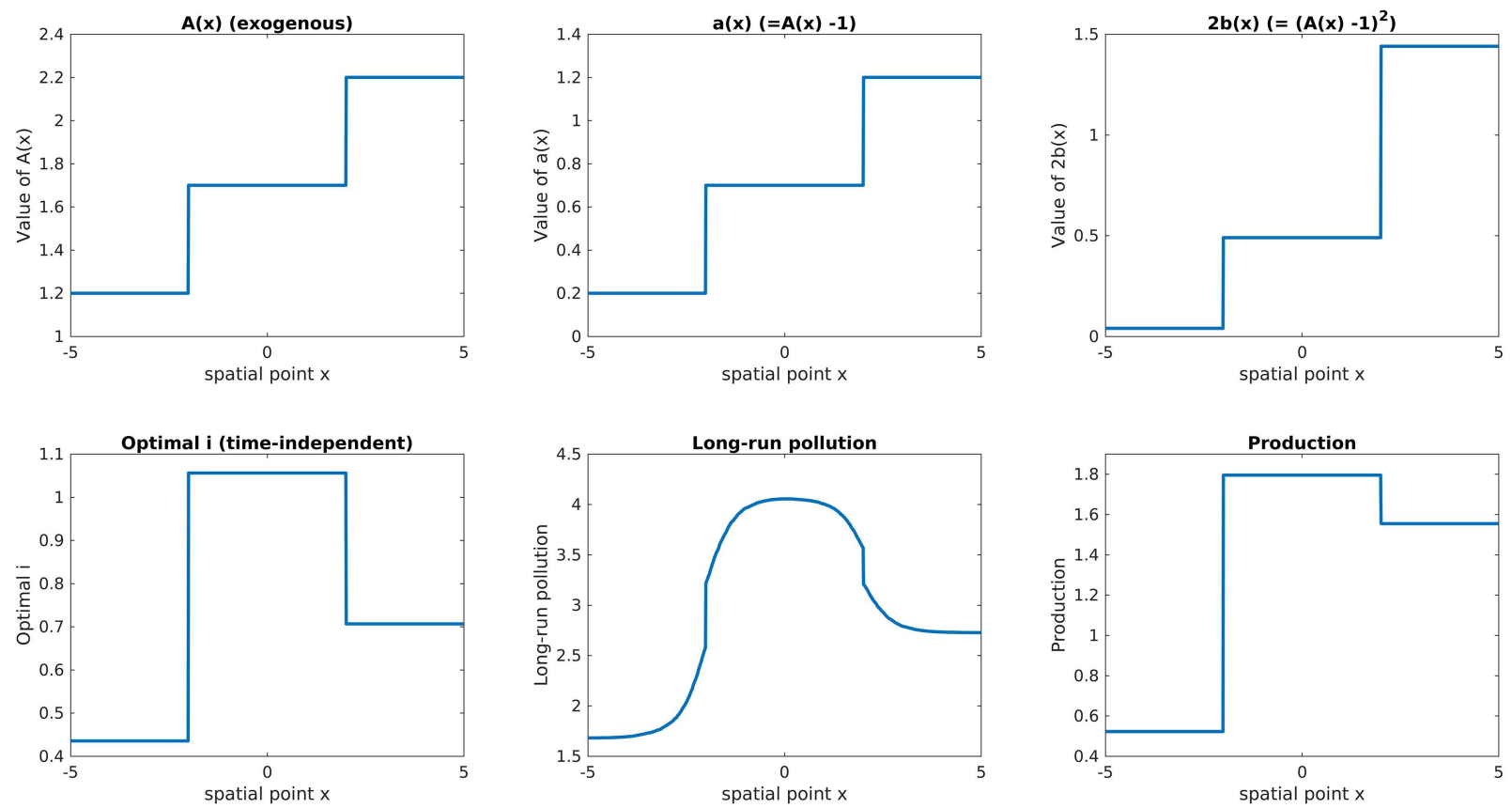

FIGURE 3. Optimal input profile, long-run pollution and production given the specified 3-steps distribution of the productivity $A(\cdot)$. Environmental awareness $w(\cdot)$ is here space-independent and equal to 0.05 , the self-cleaning capacity $\delta(\cdot)$ is space-independent and equal to $0.3, \rho=0.02$, $\sigma=\sqrt{2}$.

the right of 2 . We get in this case a spatial inverted $U$-shaped relation: a geographic environmental Kuznets curve where the Intermediate zones are the most polluted while the areas with the lowest and the highest productivity use a lower input quantity and are, at the steady state, less polluted. We use the same values for the (space-homogeneous) environmental awareness $w(\cdot)$, the (space-homogeneous) self-cleaning capacity $\delta(\cdot)$ and the parameters $\rho$ and $\sigma$ that we used in Figures 1 and 2 .

We can observe that in all the three cases (Figs. 1-3) we have a discontinuous optimal spatial distribution of the optimal $i$ (and then of the production). This fact can be easily understood looking at the form of its dependence (3.18) on the discontinuous spatial distribution of $a(\cdot)$ (also observe that, since $w(\cdot)$ and $\delta(\cdot)$ are space independent, the function $\alpha(\cdot)$ is constant in space). Conversely, the long run distribution of the pollution, whose dynamics involves the evolution of a diffusion process, is smooth.

\subsection{The impact of geographic heterogeneity in environmental awareness}

In Figure 4 we look at the effect of a geographic heterogeneity in the environmental awareness. We consider a two-steps distribution: $w(\cdot)$ equal to 0.07 in the negative half line and equal to 0.06 in the positive half-line. We can see that the impact of the heterogeneity on $w(\cdot)$ has a more straightforward effect than that of the heterogeneity in the productivity: here, not surprising, in the regions where the $w$ is higher (and then people is more averse to pollution) the agents decide to reduce the amount of input they use in order to get a smaller final pollution. To better concentrate on the effect of the environmental awareness we use in this simulation a space independent productivity $A(\cdot)$ equal to 1.3 in each point of the space and a constant self-cleaning capacity $\delta(\cdot)$ (equal to 0.05 at each point).

We can observe that, even if the distribution of the environmental awareness is simply a step function, the spatial distribution of the optimal $i$ (and consequently of the production) is smooth. Indeed in its expression at 

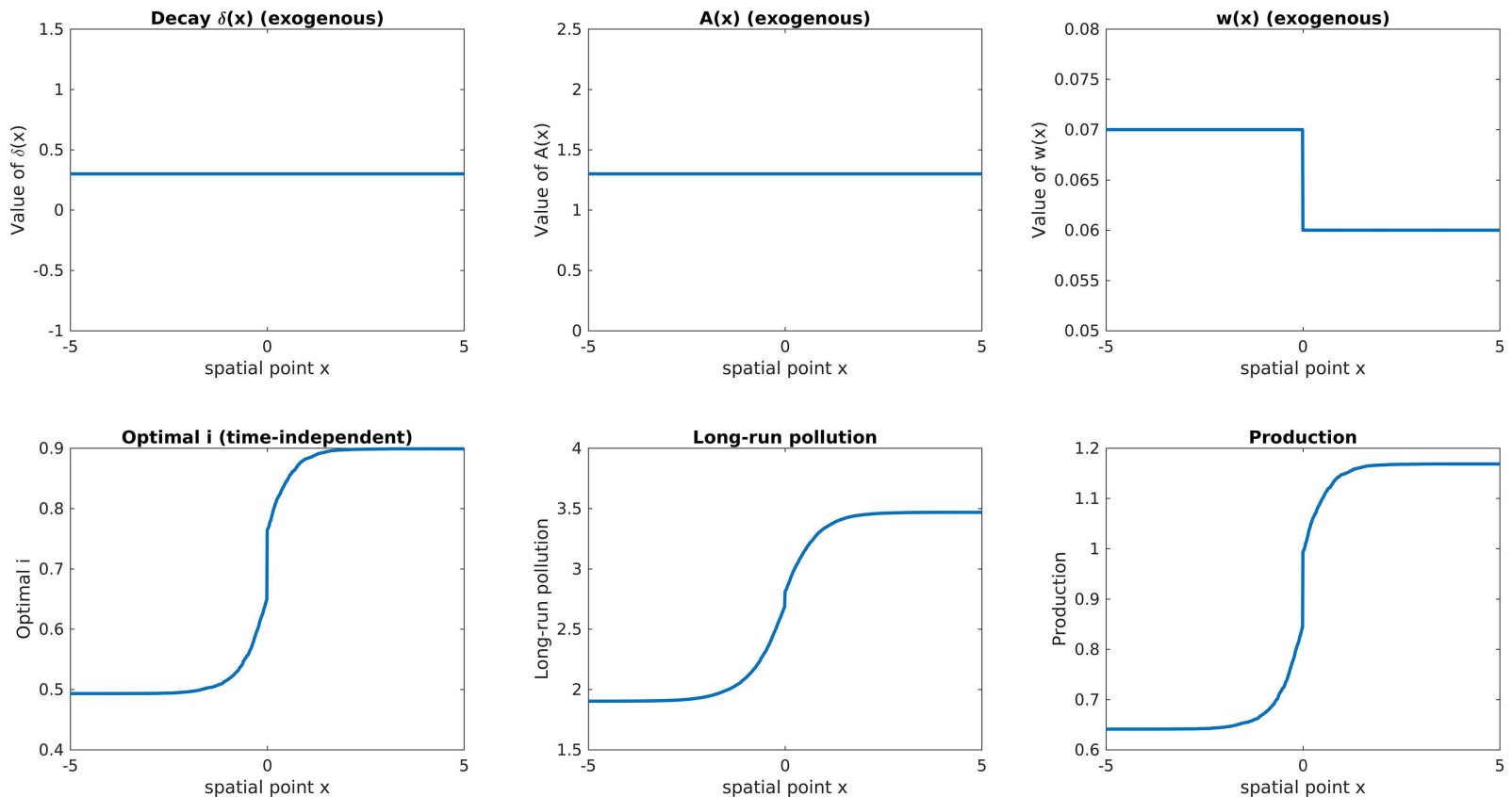

FIGURE 4. Optimal input profile, long-run pollution and production given the specified 2-steps distribution of the environmental awareness $w(\cdot)$. Productivity $A(\cdot)$ is here space-independent and equal to 1.3 , the self-cleaning capacity $\delta(\cdot)$ is space-independent and equal to $0.05, \rho=0.02$, $\sigma=\sqrt{2}$.

each spatial point (3.18) depends on the contribution of $a(\cdot)$ that is constant in space here and on $\alpha(\cdot)$ which is defined through a smoothing integral expression (4.2).

\subsection{The impact of geographic heterogeneity in nature self-cleaning capacity}

In Figures 5 and 6 we look at the effect of a geographic heterogeneity in nature self-cleaning capacity. We consider a two-steps distribution: $\delta(\cdot)$ equal to 0.03 in the negative half line and equal to 0.04 in the positive half-line. Figures 5 and 6 differ for the level of $A$ we choose. In both cases, to better underline the effect of nature self-cleaning capacity, a space independent productivity $A(\cdot)$ is chosen but we use a high value of $A$ in Figure 5 (equal to 5.5) while we use a low value (equal to 1.3) in Figure 6. The same contrasted picture we had for the impact of geographic heterogeneity in productivity reproduces here: while in both cases the areas with a higher self-cleaning capacity are those where the agents choose a higher level of input a possibly surprising result arises in terms of the long-run pollution distribution. In the case of high level of $A$ the areas with a higher self-cleaning capacity attain a lower level of long-run pollution while the opposite situation takes place in the case of a low level of $A$.

As in the case of the simulation on the impact of the distribution of the environmental awareness we can observe a smoothing effect on the spatial distributions of the optimal $i$, of the production and of the pollution.

\subsection{The simultaneous impact of geographic heterogeneities}

Several situations are possible when the mentioned heterogeneity arise at the same time but the general behavior follows a rather intuitive pattern: each of the effects we described pushes the distributions of input use, pollution and production in a certain direction and the total effect depends on which of the effects is stronger. So the general qualitatively effect is not very surprising. Still, something of non-obvious can happens 

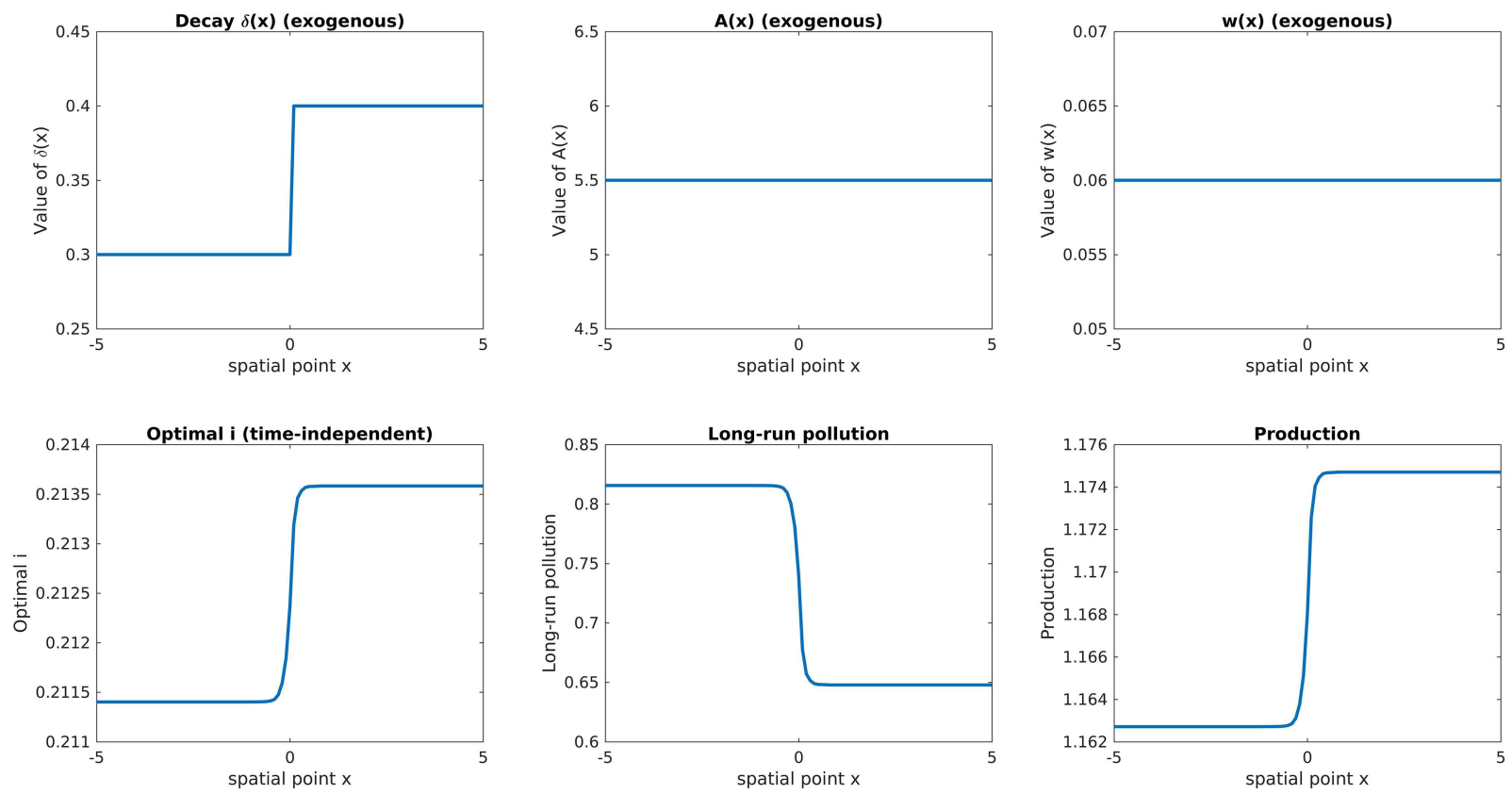

FiguRE 5. Optimal input profile, long-run pollution and production given the specified 2-steps distribution of the self-cleaning capacity $\delta(\cdot)$. Productivity $A(\cdot)$ is here space-independent and equal to 5.5 (high level of $A$ ), the environmental awareness $w(\cdot)$ is space-independent and equal to $0.05, \rho=0.02, \sigma=\sqrt{2}$.

in boundary locations. We show this fact in Figure 7 where we look at the concomitant effect of a spatial heterogeneity in the productivity $A(\cdot)$ and of the environmental awareness $w(\cdot)$. In the figure, the level of $A(\cdot)$ is equal to 1.3 in the negative half-line and 1.4 in the positive half-line while the values of $w(x)$ is 0.06 for negative values and 0.07 for positive values of $x$. The self-cleaning capacity $\delta(\cdot)$ is space-independent and equal to 0.3 , $\rho=0.02$ and $\sigma=\sqrt{2}$. The values of the two-steps distributions $A(\cdot)$ and $w(\cdot)$ are chosen in order to balance the two effects for the spatial points which are far from 0 on $i$ and then to see in more detail what happens around 0 . As one could imagine a discontinuity arises, as in the cases of discontinuous $A(\cdot)$ analyzed in Section 5.1 , in the behavior of $i$ when $x$ is near 0 but something more happens indeed we can observe a significant decrease in $i$ for negative values of $x$ and a significant increase for positive values of $x$ when $x \rightarrow 0$. This fact is due to the different smoothness of the effects of the heterogeneities of $A(\cdot)$ and $w(\cdot)$ on $i$ described, respectively, in Sections 5.1 and 5.2: while the effect of the heterogeneity of $A(\cdot)$ impacts the distribution of $i$ in a clearcut way, the effect of the heterogeneity of $w(\cdot)$ is smooth and then the former is preponderant near 0 while it is balanced by the latter when $x$ is far from 0 .

\section{Conclusion}

In this paper, we have considered a canonical spatio-temporal economy using a polluting one-input technology. While the mathematical setting is linear-quadratic and autonomous, we consider a quite rich space structure. First locations differ in productivity, nature self-cleaning technology and environmental awareness. Second, pollution has a transboundary component, resulting in a PDE diffusion equation modelling. Apart from the dynamic programming apparatus, adapted to our infinite dimensional setting, we have been able to extract several interesting result. In particular, we derive a quite interesting general condition for technological progress to curb emissions. We show that optimal emissions will decrease at given location if and only if local productivity is larger than a threshold which depends both on the local pollution absorption capacity and environmental 

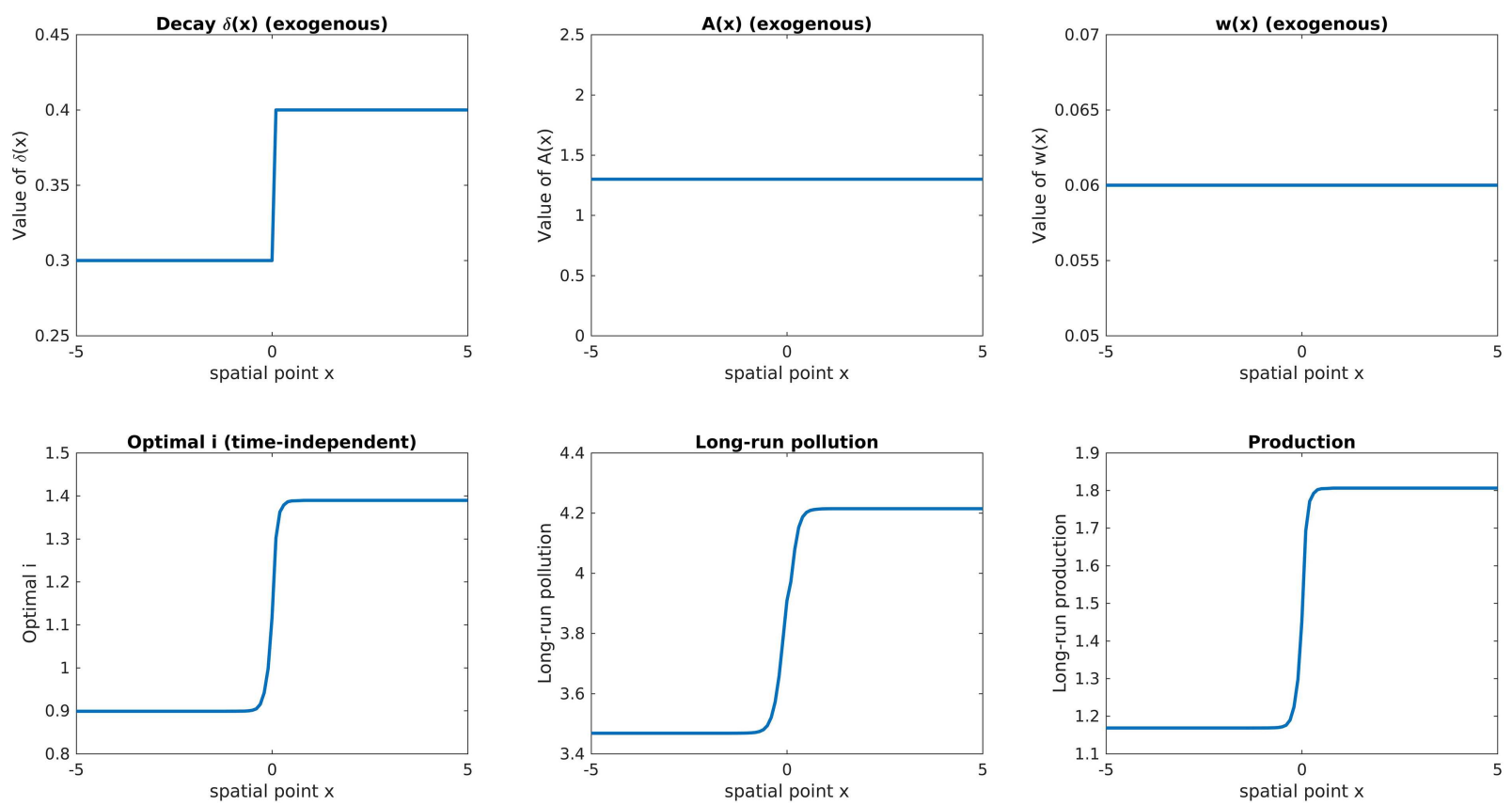

FIGURE 6. Optimal input profile, long-run pollution and production given the specified 2-steps distribution of the self-cleaning capacity $\delta(\cdot)$. Productivity $A(\cdot)$ is here space-independent and equal to 1.3 (low level of $A$ ), the environmental awareness $w(\cdot)$ is space-independent and equal to $0.05, \rho=0.02, \sigma=\sqrt{2}$.
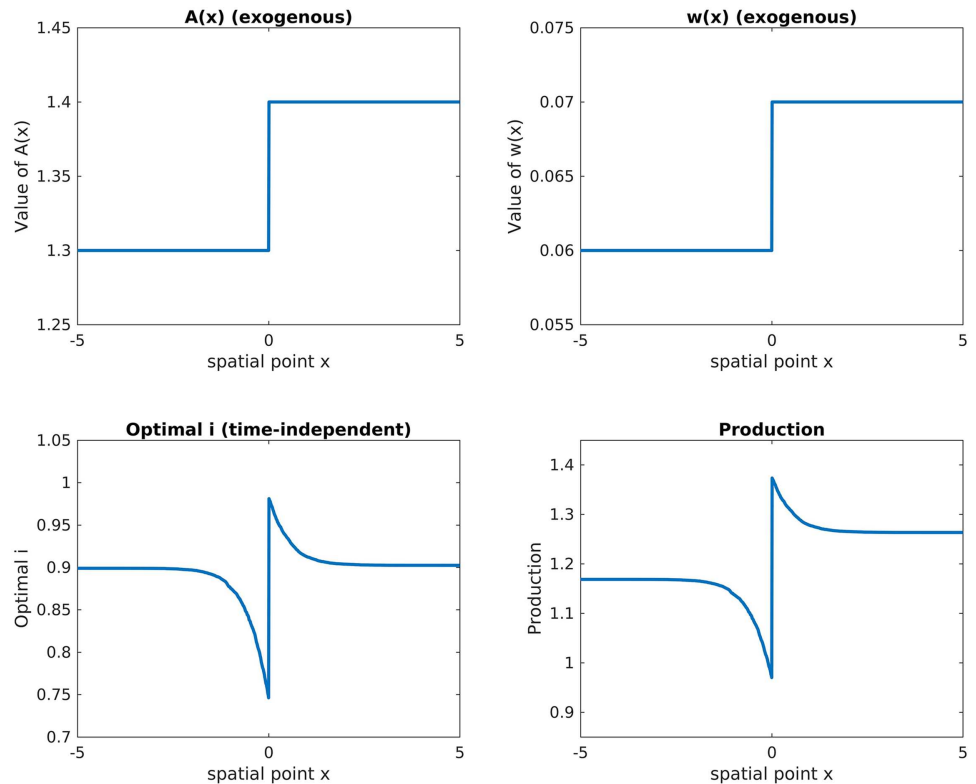

FIGURE 7. Optimal input and production profiles given the specified 2-steps distributions of the productivity $A(\cdot)$ and of the the environmental awareness $w(\cdot)$. The the self-cleaning capacity $\delta(\cdot)$ is here space-independent and equal to $0.3, \rho=0.02, \sigma=\sqrt{2}$. 
awareness. Furthermore, we have numerically explored the relationship between the spatial optimal distributions of production and (asymptotic) pollution and we have been able to detect several (geographic) Environmental Kuznets Curve cases.

Clearly the linear-quadratic setting limits the scope of some of our results. In particular, it prevents optimal investment to be time-dependent and it also prevents endogenous growth. We are working on alternative specifications allowing to address the same problem in non-linear-quadratic frameworks while keeping partially the advantages of the optimization technique developed.

\section{REFERENCES}

[1] K. Arrow, P. Dasgupta, L. Goulder, G. Daily, P. Ehrlich, G. Heal, S. Levin, K.-G. Maler, S. Schneider, D. Starrett and B. Walker, Are we consuming too much? J. Econ. Perspect. 18 (2004) 147-172

[2] E. Barucci and F. Gozzi, Investment in a vintage capital model. Res. Econ. 52 (1998) 159-188

[3] E. Barucci and F. Gozzi, Optimal advertising with a continuum of goods. Ann. Oper. Res. 88 (1999) 15-29

[4] E. Barucci and F. Gozzi, Technology adoption and accumulation in a vintage capital model. J. Econ. 74 (2002) 1-38

[5] T. Bassetti, N. Benos and S. Karagiannis, $\mathrm{CO}_{2}$ emissions and income dynamics: what does the global evidence tell us? Environ. Resource Econ. 54 (2013) 101-125

[6] A. Bensoussan, G. Da Prato, M.C. Delfour and S.K. Mitter, Representation and Control of Infinite Dimensional Systems (Second Edition). Birkhäuser, Basel (2006)

[7] R. Boucekkine, C. Camacho and G. Fabbri, Spatial dynamics and convergence: the spatial AK model. J. Econ. Theory 148 (2013) 2719-2736

[8] R. Boucekkine, G. Fabbri, F. Gozzi and S. Federico, Growth and agglomeration in the heterogeneous space: a generalized AK approach. To appear in J. Econ. Geogr. (2018). Doi: 10.1093/jeg/lby041

[9] R. Boucekkine, A. Pommeret and F. Prieur, Technological vs. ecological switch and the environmental Kuznets curve. Am. J. Agric. Econ. 95 (2013) 252-260

[10] R. Boucekkine, J. Krawczyk and T. Vallée, Environmental quality versus economic performance: a dynamic game approach. Optimal Cont. Appl. Methods 32 (2011) 29-46

[11] Br H. Brezis, Functional Analysis, Sobolev Spaces and Partial Differential Equations. Springer, Berlin (2011)

[12] C. Camacho and A. Perez Barahona Land use dynamics and the environment. J. Econ. Dyn. Cont. 52 (2015) 96-118

[13] J. De Frutos and G. Martin-Herran, Spatial effects and strategic behavior in a multiregional transboundary pollution dynamic game. To appear in: J. Environ. Econ. Manag. (2017). Doi: 10.1016/j.jeem.2017.08.001

[14] SD S. Dinda, Environmental Kuznets curve hypothesis: a survey. Ecol. Econ. 49 (2004) 431-455

[15] K.J. Engel and R. Nagel, One-parameter Semigroups for Linear Evolution Equations. Springer, Berlin (1995)

[16] S. Faggian and F. Gozzi, On the dynamic programming approach for optimal control problems of PDEs with age structure. Math. Popul. Stud. 11 (2004) 233-270

[17] G. Kallis, In defense of degrowth. Ecol. Econ. 70 (2011) 870-880

[18] I. Karatzas and S. Shreve, Brownian Motion and Stochastic Calculus. Springer Berlin (1988)

[19] LTL D. La Torre, D. Liuzzi and S. Marsiglio, Transboundary pollution externalities: think globally, think locally? (2018).

[20] A. Lunardi, Analytic Semigroups and Optimal Regularity in Parabolic Problems. Birkhäuser, Basel (1995)

[21] F. Prieur, The environmental Kuznets curve in a world of irreversibility. Econ. Theory 40 (2009) 57-90

[22] I. Schumacher and B. Zou, Pollution perception: a challenge for intergenerational equity. J. Environ. Econ. Manag. 55 (2008) 296-309

[23] R. Sengupta, $\mathrm{CO}_{2}$ emission-income relationship: policy approach for climate control. Pacific Asia J. Energy 7 (1997) 207-229

[24] D. Stern, The rise and fall of the environmental Kuznets curve. World Dev. 32 (2004) 1419-1439

[25] N. Stokey, Are there limits to growth? Int. Econ. Rev. 39 (1999) 1-31 